\title{
Neural representation of temporal duration: coherent findings obtained with the "lossy integration" model
}

\author{
Olga V. Sysoeva ${ }^{1,3 *}$, Marc Wittmann ${ }^{2}$ and Jiří Wackermann ${ }^{2}$ \\ 1 Washington University School of Medicine, Saint Louis, MO, USA \\ 2 Department of Empirical and Analytical Psychophysics, Institute for Frontier Areas of Psychology and Mental Health, Freiburg i. Br., Germany \\ 3 Institute of Higher Nervous Activity and Neurophysiology, Russian Academy of Sciences, Moscow, Russia \\ *Correspondence: sysoevao@psychiatry.wustl.edu
}

Establishing links between experimental data, their models, and the neural substrates presents a permanent challenge for research in timing and time perception. This applies particularly to the problem of internal representation of temporal duration and its neural implementation. In this short communication we will report on progress achieved with the "lossy integration" model (also known as "klepsydra" model; Wackermann and Ehm, 2006) in interpretation of time perception data in the context of neurophysiological and neurobiological findings.

In the pacemaker-gate-accumulator model (Zakay and Block, 1997), which is still considered as the standard model in the literature (Grondin, 2010), temporal durations are internally represented by cumulative pulse counts,

$$
A=t \cdot f
$$

( $t$ is the interval duration, $f$ the effective pulse train frequency, and $A$ is the number of pulses accumulated in the counter). Consequently, all variations in timing behavior or in a time perception task response can be accounted for by a change of the frequency $f$ of pulses entering the counter. This gives the model its apparent elegance and universality, but is also its main weakness. Since the two hypothetical components, pacemaker and gate, are arranged serially, it is impossible to disentangle their effects. The effective frequency $f$ may vary due to a change of the pacemaker fundamental frequency $f_{\mathrm{p}}$ (e.g., in response to organismic, physiological factors), or due to a change of the gate throughput $g$ (e.g., attentional, cognitive effects):

$$
f=f_{\mathrm{P}} \cdot g
$$

(where $g$ is a real number in the range from 0 to 1 ).

By contrast, in the "klepsydra" model, (Wackermann and Ehm, 2006), temporal durations are represented by states of a lossy integrator; written in a differential form

$$
\frac{d A}{d t}=f-\kappa \cdot A
$$

The inflow rate $f$ corresponds to the pulse train frequency in the "standard" model (that the states are here continuous, rather than discrete quantities, is unimportant). The outflow rate, however, is determined by the momentary state of the accumulator $A$ and a proportionality factor $\kappa$. Therefore, there are two loci of possible effects on internal time representation, the inflow and the outflow. The inflows can be studied only in relative terms, e.g., as inflow ratios between different experimental conditions; however, the loss rate $\kappa$ can be determined numerically in given physical units $\left(\mathrm{sec}^{-1}\right)$.

To apply the model to the two tasks mostly used in our experimental studies - duration reproduction and duration discrimination in the supra-second range - we assume two such inflow-outflow units, each one allocated to one of the two temporal intervals to be compared. [Hence the name "dual klepsydra model" under which the model is known (Wackermann and Ehm, 2006).] Numerical procedures are available for estimating the value of $\kappa$ directly from (individual or group-based) response functions in the reproduction task (Wackermann and Ehm, 2006), or indirectly, from points of subjective indifference determined by psychometric functions fitted to the data from the discrimination task (Wackermann and Späti, 2006). The lossy character of internal time representation is revealed by the progressive shortening of the reproduction response, or by the presentation order effect in the discrimination response (generally known as "subjective shortening" of past durations).

Recent experiments supported the notion of the "loss rate" parameter $\mathrm{K}$ as a stable individual characteristic of the subject, evidenced by the high test-retest reliability of the parameter $\kappa$ obtained from duration discrimination data (Sysoeva et al., 2010) and duration reproduction data. Moreover, it was shown (Sysoeva et al., 2010) that carriers of genotypic variants related to the activity of the serotonergic (5-HT) transmitter system significantly differ in the "loss rate" parameter $\kappa$. These results suggest genetic determination of dynamic parameters of neural representation of time. Higher values of $\kappa$ were found for the carriers of genotypes characterized by higher potential for 5-HT transmission: (1) lower 5-HT reuptake, known for the 5-HTTLPR SS polymorphism compared with LL, (2) lower 5-HT degradation, described for the "low expression" variant of MAOA VNTR gene compared with "high expression" variant, and (3) higher 5-HT2a receptor density, proposed for the TT polymorphism of 5-HT2a T102C gene compared with CC. Also, they fit well with findings in studies on effects of psychotropic substances affecting the serotonin subsystem. In a double-blind, placebo-controlled study, psilocybin - a serotonin (5-HT) 2A/1A receptor agonist significantly increased parameter $\kappa$ which is indicative of a higher "loss rate" of duration representation, observable by a stronger under-reproduction of temporal intervals (Wackermann et al., 2008). These convergent findings suggest an action path from 5-HT activity-related genes, via activity of 5-HT in the brain, to time perception. The psychopharmacological data also indicate that although the loss rate parameter is genetically determined, it can be temporally modified by influencing the 5-HT system.

In a fMRI study, it was shown that parameter $\kappa$ and the degree of self-rated impulsivity were associated with brain activation during the reproduction phase of the duration reproduction task; the activated brain areas were those related to motor execution as well as to the "core control network." In particular, activation in these regions was positively correlated with the "loss rate" parameter $\kappa$ (i.e., more pronounced 
under-reproduction of intervals), and with the subject's degree of impulsivity (Wittmann et al., 2011). During the encoding of duration in the reproduction task brain activation within bilateral posterior insula showed an accumulating pattern over time which peaked at the end of the interval (Wittmann et al., 2010). Based on the knowledge about insular cortex functioning it has been suggested that the integration of ascending body signals forms the basis for the representation of duration (Craig, 2009; Wittmann, 2009). This hypothesis is supported by recent observations of an association between the decrease of heart-beat frequency - indicative of an increase in parasympathetic activity - during the encoding of duration in individuals performing the duration reproduction task (Meissner and Wittmann, 2011). Interpreting these empirical findings in term of the klepsydra model, the flux of bodily signals into the posterior insular cortex could be interpreted as constituting the inflow component of the model. A more widespread network encompassing the "core control network" (Cole and Schneider, 2007) which would be associated with maintaining the representation of duration over time, can be related to the outflow component of the model, thus representing the "loss rate" of the leaky accumulator.

The work reported above focused on effects of the loss component of the klepsydra model, as the "subjective shortening" is a striking phenomenon seen in duration reproduction or duration discrimination data in the supra-second region. Since these effects are omnipresent in the data (whether they are subject matter of study or not) they have to be taken into account to distinguish net effects of experimental manipulations. The klepsydraic model not only disentangles the inflow (accumulation) and outflow (loss) effects conceptually, but it also allows to separate these effects operationally. An example of this analytic strategy is given in the study of brightness-duration interaction in a duration discrimination task (Wackermann and Meyer-Blankenburg, 2009), where the net effect caused by the stimulus variation is superimposed on the main stimulus-independent effect of subjective shortening. Similar strategies should be applicable in studies intending to manipulate the hypothetical "inflows" by varying somatosensory or proprioceptive stimuli to test the "bodily signals flux" hypothesis.

Summarizing: in the reported studies effects of natural variations or experimental manipulations on time perception were evaluated by means of a simple "lossy integration" model, which conceptually distinguishes between two components of the mechanism underlying internal representation of temporal durations: accumulation of internal "inflow" in the integrator, and a parallel "loss" of accumulated representation ("outflow"). It is suggested that the inflow is primarily derived from the ongoing stream of intero- and proprioceptive neural signals, while the outflow is related to low-level (synaptic?) mechanisms of neural signals transfer. Converging findings on neurophysiological or neurochemical effectors or correlates of time perception provide cumulative evidence for these working hypotheses. Therefore, we wish to draw the attention of the research community to this fruitful methodology, which promises to obtain new insights into human timing and time perception in experimental research as well as in studies of clinical populations.

\section{REFERENCES}

Cole, M. W., and Schneider, W. (2007). The cognitive control network: integrated cortical regions with dissociable functions. Neuroimage 37, 343-360.

Craig, A. D. (2009). Emotional moments across time: a possible neural basis for time perception in the anterior insula. Philos. Trans. R. Soc. Lond., B, Biol. Sci. 364, 1933-1942.

Grondin, S. (2010). Timing and time perception: a review of recent behavioral and neuroscience findings and theoretical directions. Atten. Percept. Psychophys. 72, 561-582.

Meissner, K., and Wittmann, M. (2011). Body signals, cardiac awareness, and the perception of time. Biol. Psychol. 86, 289-297.

Sysoeva, O. V., Tonevitsky, A., and Wackermann, J. (2010). Genetic determinants of time perception mediated by the serotonergic system. PLoS ONE 5, e12650. doi: 10.1371/journal.pone.0012650

Wackermann, J., and Ehm, W. (2006). The dual klepsydra model of internal time representation and time reproduction. J. Theor. Biol. 239, 482-493.

Wackermann, J., and Meyer-Blankenburg, N. (2009). "Differential effect of stimulus brightness in visual duration discrimination," in Fechner Day 2009, eds M. A. Elliott, S. Antonijevic, S. Berthaud, P. Mulcahy, B. Bargary, C. Martyn, and H. Schmidt (Galway: International Society for Psychophysics), 459-464.

Wackermann, J., and Späti, J. (2006). Asymmetry of the discrimination function for temporal durations in human subjects. Acta Neurobiol. Exp. (Wars) 66, 245-254.

Wackermann, J., Wittmann, M., Hasler, F., and Vollenweider, F. X. (2008). Effects of varied doses of psilocybin on time interval reproduction in human subjects. Neurosci. Lett. 435, 51-55.

Wittmann, M. (2009). The inner sense of time. Philos. Trans. R. Soc. Lond., B, Biol. Sci. 364, 1955-1967.

Wittmann, M., Simmons, A. N., Aron, J., and Paulus, M. P. (2010). Accumulation of neural activity in the posterior insula encodes the passage of time. Neuropsychologia 48, 3110-3120.

Wittmann, M., Simmons, A. N., Flagan, T., Lane, S. D., Wackermann, J., and Paulus, M. P. (2011). Neural substrates of time perception and impulsivity. Brain Res. 1406, 43-58.

Zakay, D., and Block, R. A. (1997). Temporal cognition. Curr. Dir. Psychol. Sci. 6, 12-16.

Received: 28 July 2011; accepted: 29 July 2011; published online: 12 August 2011.

Citation: Sysoeva OV, Wittmann M and Wackermann J (2011) Neural representation of temporal duration: coherent findings obtained with the "lossy integration" model. Front. Integr. Neurosci. 5:37. doi: 10.3389/fnint.2011.00037 Copyright ( 2011 Sysoeva, Wittmann and Wackermann. This is an open-access article subject to a non-exclusive license between the authors and Frontiers Media SA, which permits use, distribution and reproduction in other forums, provided the original authors and source are credited and other Frontiers conditions are complied with. 